\section{More than One Ever Wanted to Know about X-Ray Detectors Part VI: Alternate Semiconductors for Detectors}

\author{
Mark W. Lund, Ph.D., MOXTEK, Inc.
}

$X$-ray spectrometers give the capability to determine chemical element composition in electron microscopes. The semiconductor with the most experience as an X-ray detector is silicon. Silicon is the most highly developed material on earth, and has a lot of good things going for it, but for some applications we crave something with other good properties. For example, for room temperature detectors it would be best to have a semiconductor with a wider band gap. For higher resolution it would be better to have a semiconductor with a smaller band gap. For these reasons a number of other serniconductors have been developed as $\mathrm{x}$-ray detectors. In this article I will talk about narrow band gap semiconductors. Next time I will discuss large band gap semiconductors.

As I discussed in Part II, the resolution of energy dispersive detectors is limited by the Poisson-Fano statistics of the charge generation process. The more charge generated by an $\mathrm{x}$-ray the smaller the noise from this source. Thus one of the trends in EDX is to move to semiconductors with smaller band gaps. The natural place to go from silicon is germanium, which has 1.3 times the charge generation. The Fano factor of germanium also seems to be about $20 \%$ lower than silicon. These two factors give germanium about a $25 \%$ resolution advantage over silicon without considering the electronic noise, which will dominate at low energies.
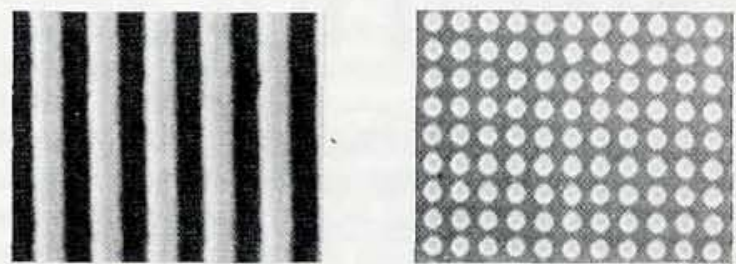

\section{Submicron Calibration Standards}

$0.3 \mu \mathrm{m}$ and $0.7 \mu \mathrm{m}-1 \mathrm{D}$ and $2 \mathrm{D}$

\section{Original Gratings}

Tungsten over Polymer on Silicon

\section{Accurate}

$3 \sigma$ variation $\pm 3 \%$ over $3 \mathrm{~mm} \times 4 \mathrm{~mm}$ area

Durable

over 1,000,000 new measurement sites per sample

Easy to Use

Rated 4.4 out of 5.0 at labs like yours

Versatile

$3 \mathrm{~mm} \times 4 \mathrm{~mm}$ silicon chip can be mounted anywhere

Inexpensive - Especially NOW! All 4 types: $\$ 275$ each* $^{*}$

\section{Available Today!}

Ask for the New MOXTEK Calibration Standard from your favorite microscopy supplier or

\section{MOXTEK, Inc. (801)225-0930}

\section{Try one now Risk Free*}

If you don't like it send it back for a full refund

*This Special Introductory Offer ends September 15, 1995.
Germanium was used as an EDX detector from the very beginning of EDX and has always been the preferred material for gamma ray detection. Due to technical problems it has not shined as an $\mathrm{x}$-ray detector material until recently. It has several advantages besides the noise. In the "old days" germanium was lithium drifted to make $\mathrm{Ge}$ (Li) detectors. In the modern age germanium can be made purer than silicon, and so does not have to be lithium drifted. It has much higher stopping power than silicon. It has higher mobility (and mobility-lifetime product) which allows the charge to be collected more completely. Germanium detectors have been used extensively in gamma ray spectroscopy due to this ability to collect charge over large volumes and their high stopping power. High resolution germanium $x$-ray detectors have only been available very recently due to some problems that germanium presents.

Germanium has also had its drawbacks. One of the worst is a by-product of its high stopping power. At Al $K \alpha 25 \%$ of the $\mathrm{x}$-rays are stopped within the first 0.1 micron. This means that the detector "dead layer" must be very small or partially collected charge will widen the $x$-ray peaks. The electric fields must also be high to prevent part of the charge from diffusing to the top surface of the detector where they will recombine. Germanium is sensitive to infrared light, and so requires a separate cooled window in the cryostat to block this light.

As I discussed in Part III, a common artifact in EDS is the escape peak, which happens when a signal $x$ ray loses some of its energy by creating a fluorescent $x$-ray in the detector. Most of these secondary $x$ rays will be reabsorbed by the detector. However, some of the secondary $x$-rays escape from the detector, leaving a deficit in the signal and producing a ghost peak. Silicon has an absorption edge at $1.84 \mathrm{keV}$, and its escape peaks are $1.74 \mathrm{keV}$ below each parent peak. Germanium has no escape peaks up to its absorption edge at $11.1 \mathrm{keV}$. (There is a theoretical possibility of an escape peak from the $\mathrm{Ge} L \alpha$, but this peak has not been seen.) This is great if there are no x-rays coming into the detector with energy greater than $11.1 \mathrm{keV}$. If there are then you get an unexpected bonus of two escape peaks $1.1 \mathrm{keV}$ apart, one from the $\mathrm{Ge} K \alpha$ and one from the Ge K $\beta$. In a complicated spectrum this could be quite a mess: for zirconium the escape peaks from both $\mathrm{Zr} \mathrm{K} \alpha$ and $\mathrm{Zr} \mathrm{KB}$ are all resolved. In addition the higher fluorescence yield of germanium makes the ratio of escape count to $K \alpha$ ten to a hundred times higher than silicon. This is why computers were invented!

If germanium, with its smaller band gap, can give better resolution how about other small-band-gap semiconductors? HgCdTe has an adjustable band gap clear down to zero, as do some other ternary (three-element) compounds. Theoretically these materials could be used to give much better resolution. These narrow band gap semiconductors have been developed for infrared detectors and are very successful. However, they have a lot of leakage current due to the difficulty in preparing ultra-high quality crystals from ternary compounds. Infrared detector systems have tons of signal which can easily make up for the leakage current, but $\mathrm{x}$-ray detectors must detect pulses of only a few dozen electrons. There is always hope that narrow-band-gap semiconductors will improve in quality to the point that they can be used.

I wonder how many researchers have wished, as I often do, to travel back in time to the Creation of the Universe and make a simple request. My dream is to have three more chemical elements in column IVA in the periodic table--one inserted between diamond and silicon, one between silicon and germanium, and one between germanium and tin. Having these elements would give the $x$-ray detector business a big boost! I would gladly give up erbium, thulium and ytterbium for silicon-plus, germanium-plus, and tin-plus! Why? As you go down column IVA the bandgap decreases from diamond $(5.5 \mathrm{eV}$ ) to tin (exactly zero). Single -element crystals are much easier to make perfect than are compounds. An intermediate between germanium and tin would have a narrow band gap, high stopping power, and excellent crystal perfection, and would make a great detector. There are similar good reasons for making detectors from silicon-plus or germanium-plus.

One approach to this (without a time machine) is to make compounds or alloys of the column IVA elements. In most cases a compound or alloy of two semiconductors will have a band gap intermediate between the two constituents. If we could just lift the band gap of tin a couple of millielectron volts... Unfortunately tin does not make compounds or alloys with carbon, silicon or germanium. This leaves us without a column IVA (i.e. covalently bonded) possibility for a band gap lower than germanium. Likewise carbon and germanium are incompatible, but such combinations as silicon carbide or silicon-germanium alloys have interesting properties that I will discuss in the next article. 
